\title{
Advanced cone-beam CT venous angiographic imaging
}

\author{
Visish M. Srinivasan, MD, ${ }^{1}$ Gouthami Chintalapani, PhD, ${ }^{2}$ Edward A. M. Duckworth, MD, ${ }^{3}$ and \\ Peter Kan, MD'
}

\begin{abstract}
${ }^{1}$ Department of Neurosurgery, Baylor College of Medicine, Houston, Texas; ${ }^{2}$ Siemens Medical Solutions, Hoffman Estates, Illinois; and ${ }^{3}$ Northwest Neurosurgical Associates, Boise, Idaho
\end{abstract}

\begin{abstract}
OBJECTIVE The evaluation of the venous neurovasculature, especially the dural venous sinuses, is most often performed using MR or CT venography. For further assessment, diagnostic cerebral angiography may be performed. Three-dimensional rotational angiography (3D-RA) can be applied to the venous system, producing 3D rotational venography (3D-RV) and cross-sectional reconstructions, which function as an adjunct to traditional 2D digital subtraction angiography.

METHODS After querying the database of Baylor St. Luke's Medical Center in Houston, Texas, the authors reviewed the radiological and clinical data of patients who underwent 3D-RV. This modality was performed based on standard techniques for 3D-RA, with the catheter placed in the internal carotid artery and a longer $\mathrm{x}$-ray delay calculated based on time difference between the early arterial phase and the venous phase.
\end{abstract}

RESULTS Of the 12 cases reviewed, 5 patients had neoplasms invading a venous sinus, 4 patients with idiopathic intracranial hypertension required evaluation of venous sinus stenosis, 2 patients had venous diverticula, and 1 patient had a posterior fossa arachnoid cyst. The x-ray delay ranged from 7 to 10 seconds. The 3D-RV was used both for diagnosis and in treatment planning.

CONCLUSIONS Three-dimensional RV and associated cross-sectional reconstructions can be used to assess the cerebral venous vasculature in a manner distinct from established modalities. Three-dimensional RV can be performed with relative ease on widely available biplane equipment, and data can be processed using standard software packages. The authors present the protocol and technique used along with potential applications to venous sinus stenosis, venous diverticula, and tumors invading the venous sinuses.

https://thejns.org/doi/abs/10.3171/2017.2.JNS162997

KEY WORDS digital subtraction angiography; 3D; cone-beam CT; sinus stenosis; venous diverticulum; DSA; angiogram; vascular disorders

$\mathrm{T}$ HREE-DIMENSIONAL rotational angiography (3D-RA) has now become mainstream in the evaluation of the arterial vasculature in the angiography suite. It can be performed on flat-panel fluoroscopy equipment using 1 or 2 rotational acquisitions, each lasting $4-5$ seconds. Three-dimensional RA reconstruction uses cone-beam CT (CBCT) reconstruction principles and can provide both angiographic 3D visualization as well as CT-like multiplanar reformatted (MPR) images. The shorter scan time for 3D-RA acquisition allows easy application in awake patients and enables the operator to study neurovasculature in isolation as well as in relation to the surrounding structures. These images are distinct from the CBCT im- ages with which neurointerventionalists are familiar, that are based on longer, 20-second acquisitions. The long acquisitions limit their application in venography, because it is difficult to capture opacification of the venous system in isolation over that time span.

The current literature and application of 3D imaging (3D-RA and CBCT) has been focused on arterial vasculature. However, there are several pathologies of the venous system to which these techniques could be applied, augmenting the information obtained from traditional 2D digital subtraction angiography (DSA), MR venography (MRV), and CT venography (CTV). ${ }^{1}$ Three-dimensional rotational venography (3D-RV) can be applied in the eval-

ABBREVIATIONS CBCT = cone-beam CT; CTV = CT venography; DSA = digital subtraction angiography; ICA = internal carotid artery; MPR = multiplanar reformatted; MRV = MR venography; SSS = superior sagittal sinus; 3D-RA = 3D rotational angiography; 3D-RV = 3D rotational venography.

SUBMITTED December 1, 2016. ACCEPTED February 24, 2017.

INCLUDE WHEN CITING Published online August 18, 2017; DOI: 10.3171/2017.2.JNS162997. 
uation of venous anatomy as an adjunct to 2D DSA by extending the concept of 3D-RA acquisition.

CBCT-based imaging has been applied for venous and arteriovenous lesions. ${ }^{3,12,14}$ However, the methodology used to visualize the venous system without the arterial phase is slightly different. One method, described in a recent case report to visualize dural arteriovenous fistulas, is via a transvenous injection. ${ }^{15}$ Techniques for CBCT-based venous imaging have been described for other applications, such as adrenal vein sampling, ${ }^{2}$ liver embolization, ${ }^{8}$ and gastric varices. ${ }^{7}$ Nishio et al. ${ }^{11}$ and later Mitsuhashi et al. ${ }^{10}$ described 3D-RV performed using a transarterial route and X-ray delay. We used similar methods in our series, but we relied on the advancements in flat-panel detector technology and postprocessing software that was not available at the time of their studies (2004 and 2007).

In this paper, we present a method to acquire 3D-RV images in an angiographic suite for evaluation of venous anatomy. We describe a retrospective series of patients who underwent advanced 3D-RV. In addition to visualizing cerebral veins, a 3D-RV acquisition can also produce cross-sectional MPR images, similar to CTV. These reconstructed images offer the benefits of localization of vascular structures with respect to soft tissue and osseous structures. In effect, 3D-RV images are very similar in functionality to the 3D-RA/CBCT images with which most neurointerventionalists are familiar and can be performed with ease in both awake and sedated patients with faster acquisition time.

While these techniques were developed using a particular C-arm and associated software (Artis zee biplane, Siemens AG), the concept is generalizable to other platforms.

\section{Methods}

After obtaining appropriate consents, we reviewed our institutional endovascular database for patients who underwent advanced venography (3D-RV with or without MPR). All procedures were performed at Baylor St. Luke's Medical Center in Houston, Texas. Institutional review board approval was obtained for this retrospective study of patients who underwent 3D-RV, although all imaging was performed for clinical evaluation and not for the purposes of research.

\section{Imaging}

All angiography procedures were performed in an angiographic suite equipped with a flat-panel biplane system (Artis zee biplane, Siemens AG). C-arm CT-based 3D venography was performed in 12 patients. A 5-Fr catheter was placed in the internal carotid artery (ICA), and 3D rotational angiographic imaging was performed after administration of contrast medium. The imaging protocol used was a 5-second DSA protocol (5-second rotation time, 2 rotations, $200^{\circ}$ angular coverage, 133 frames/rotation, $70 \mathrm{kV}, 0.36 \mathrm{mGy} /$ frame), and the injection protocol used was a $2.5-\mathrm{ml} / \mathrm{sec}$ injection rate for a total volume of $18 \mathrm{ml}, 0$-second injection delay, and 7- to 10-second x-ray delay. The x-ray delay for 3D-RV acquisition was determined from the 2D DSA images as explained below.

\section{Acquisition Timing}

Three-dimensional RV images were obtained using a longer x-ray delay compared with typical 3D-RA acquisition. This delay corresponds to the time it takes for the contrast bolus to opacify the venous structures via transarterial injection. The length of delay was determined by performing a traditional DSA injection via the ICA, based on standard injection. ${ }^{4}$ From this, the point of optimal venous opacification was identified. The x-ray delay was the difference between initial arterial opacification and the venous phase. As these techniques use back projection to reconstruct the vasculature, it is important that the veins/sinuses of interest are opacified throughout the time of acquisition.

\section{Image Processing}

Three-dimensional RV projection images were automatically transferred to the associated clinical 3D workstation (Leonardo, Siemens AG) and reconstructed. Two 3D reconstructions were performed: 1) subtracted rotational angiographic reconstruction using HU Normal kernel to visualize the vasculature, and 2) CTV-like MPR using HU Sharp kernel to visualize both vasculature and surrounding anatomical structures (bone, parenchyma) as shown in Fig. $1 \mathrm{~B}$ and $\mathrm{C}$. The reconstructions are performed with a $512 \times 512$ voxel size with an inherent image resolution of $0.36 \mathrm{~mm}^{3}$.

In several cases, a "3D-3D venous fusion" was performed from 2 separate 3D-RV data sets. This technique was described previously by our group for $3 \mathrm{D}-\mathrm{RA}^{5}$ and was applied here to optimize the evaluation of the venous system where relevant.

\section{Results}

Retrospective review of the institutional databases revealed a total of 12 cases in which venous-phase advanced angiography was performed. The demographic data for these patients is shown in Table 1. In total, there were 7 cases in which 3D-RV or CTV-like images were used for diagnostic purposes and 5 cases in which the modality was used during treatment or posttreatment evaluation. There were 4 cases of venous sinus stenosis (3 of which were stented), in which the patients had symptomatology of idiopathic intracranial hypertension (pseudotumor cerebri). Two patients had venous diverticula/aneurysms causing pulsatile tinnitus, both of whom were treated. Five patients had dural-based masses that invaded either the superior sagittal sinus (SSS) or the transverse sinus, causing obstruction or occlusion. The average additional radiation dose for the 3D-RV was $57.8 \pm 20.7 \mathrm{mGy}$.

\section{Illustrative Cases \\ Case 4: Venous Sinus Stenosis}

A 62-year-old woman with a medical history of obesity, diabetes, hyperlipidemia, and hypothyroidism presented with left-sided headache, imbalance, vertigo, and decreased cognition. Workup for idiopathic intracranial hypertension was performed, and the results revealed an elevated opening pressure on lumbar puncture; MRV showed an absence of the right jugular vein, possibly relat- 

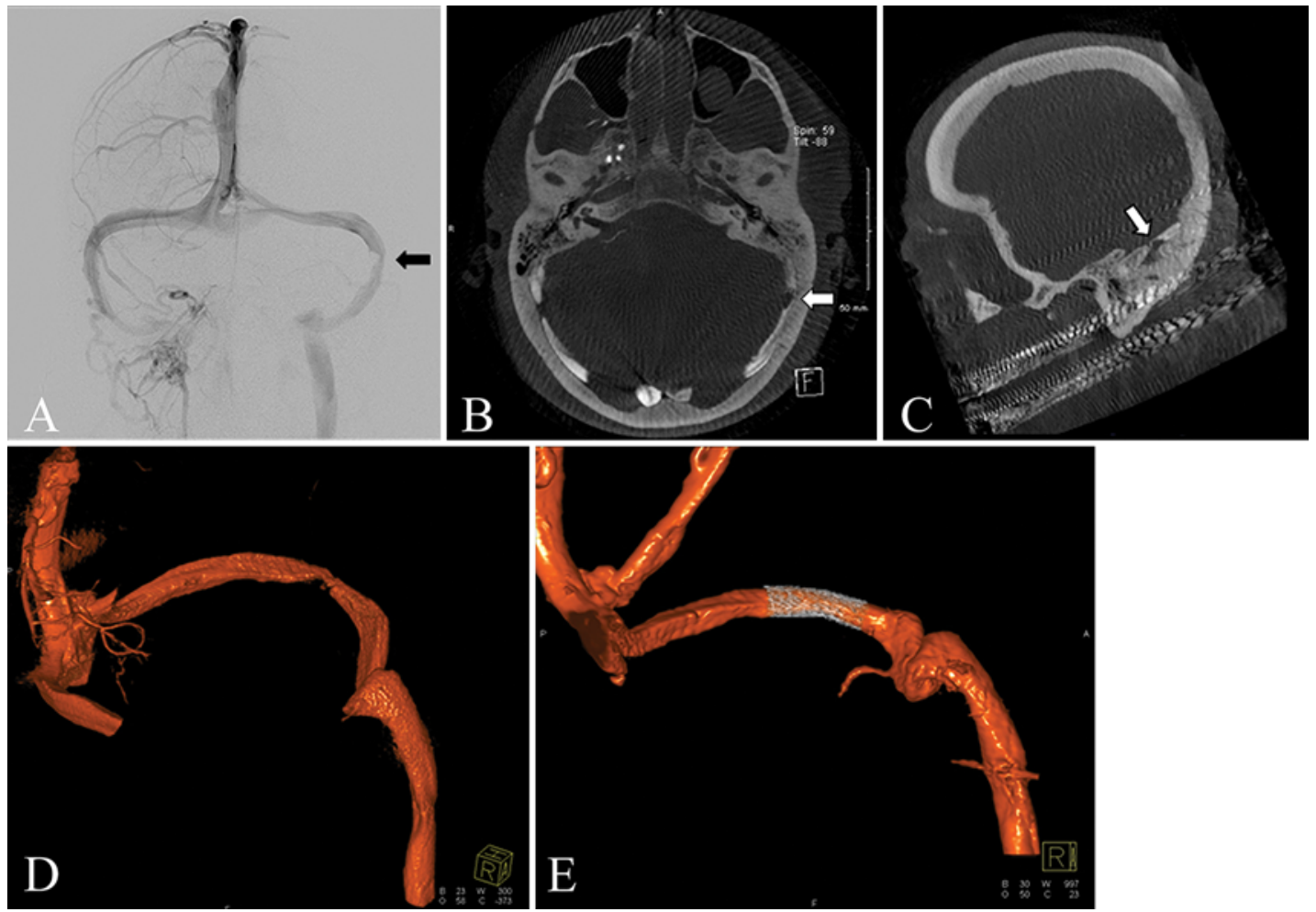

FIG. 1. Case 4. A: Anteroposterior DS angiogram showing moderate focal stenosis (arrow) that is poorly characterized. B and C: Axial and sagittal cross-sectional "CTV-like" reconstructions acquired based on an 8-second x-ray delay determined from the DS angiogram, showing severe focal stenosis of the lateral transverse sinus (arrows). D: The 3D-RV image shows the same focal stenosis. E: The stenosis was successfully treated with stenting. Figure is available in color online only.

ed to resection of a right vagus nerve schwannoma. There also seemed to be a focal stenosis of the left transversesigmoid junction.

The patient was referred for angiographic evaluation with venous sinus manometry and possible treatment. Two-dimensional angiography demonstrated only mild stenosis (Fig. 1A). However, CTV-like reconstructions and 3D reconstruction demonstrated far more severe (> $90 \%$ ) stenosis (Fig. 1B-D). The patient was treated with the placement of a $6 \times 20-\mathrm{mm}$ Precise stent (Cordis), with correction of stenosis (Fig. 1E).

\section{Case 12: Pulsatile Tinnitus/Venous Diverticulum}

A 29-year-old woman presented with headaches, dizziness, and right-sided, debilitating pulsatile tinnitus. She was suspected to have a venous diverticulum, which was initially seen on CT through bony erosion (Fig. 2C). Angiography revealed a distinct venous diverticulum arising from the junction of the right transverse sinus with the sigmoid sinus (Fig. 2A and B). The right-sided aneurysms were coil embolized with balloon assistance (Fig. 2D and E) and transvenous access was guided by the 3D-RV (Fig. 2F).

\section{Case 5: Transverse Sinus Occlusion}

A 63-year-old woman who presented with facial pain was found to have a meningioma arising from the dura under the asterion, associated with significant cerebral edema due to a suspected sinus occlusion. Cerebral angiography and venography was performed to evaluate the patency of the sinus as well as drainage of the ipsilateral vein of Labbé. There was focal occlusion of the left transverse-sigmoid junction (Fig. 3). The vein of Labbé was patent and drained into the sigmoid sinus just lateral to this focal occlusion (Fig. 3C). The patient underwent resection of the tumor. The transverse sinus was ligated during surgery just lateral to the tumor (and proximal to the drainage of the vein of Labbé into the sigmoid sinus). This allowed for resection with a dural margin, including the tentorium. The information garnered from the MPR images (Fig. 3B and C) was useful to guide the extent of resection.

\section{Case 10: Parasagittal Tumor}

A 34-year-old man presented with headaches and rightsided weakness. Further imaging revealed a dura-based, left parietal parasagittal tumor. The patency of the SSS was unclear. The patient was referred for preoperative embolization and evaluation of the sinus. Three-dimensional RV with a 9-second x-ray delay was performed. The delay was calculated based on findings on DSA after ICA injection (Fig. 4A and B). Bilateral 3D-3D venous fusion was performed for complete evaluation of the sinus, which was found to have some trace flow (Fig. 4C and D). The tumor was embolized prior to surgery and resected uneventfully. 
TABLE 1. Summary of clinical and radiological data for all patients undergoing 3D-RV

\begin{tabular}{|c|c|c|c|c|c|c|c|}
\hline $\begin{array}{l}\text { Case } \\
\text { No. }\end{array}$ & $\begin{array}{l}\text { Age } \\
\text { (yrs), } \\
\text { Sex }\end{array}$ & $\begin{array}{l}\text { Reason for } \\
\text { Angiography }\end{array}$ & Anesthesia & $\begin{array}{l}\text { Previous } \\
\text { Imaging }\end{array}$ & $\begin{array}{l}\text { Advanced } \\
\text { Venous Imaging }\end{array}$ & $\begin{array}{l}\text { X-Ray } \\
\text { Delay } \\
\text { (sec) }\end{array}$ & Outcome/Intervention \\
\hline 1 & $39, \mathrm{~F}$ & $\begin{array}{l}\text { Venous sinus } \\
\text { stenosis }\end{array}$ & GETA & CTV & $\begin{array}{l}\text { 3D-RV, bilat 3D } \\
\quad \text { fusion }\end{array}$ & $9^{*}$ & $\begin{array}{l}\text { Normal venous sinus drainage pattern w/o pressure gradient or } \\
\text { stenosis }\end{array}$ \\
\hline 2 & $29, \mathrm{~F}$ & $\begin{array}{l}\text { Venous sinus } \\
\text { stenosis }\end{array}$ & MAC & CTV & 3D-RV, MPR & $8^{*}$ & $\begin{array}{l}\text { Venous stenting; dominant It transverse-sigmoid sinus, w/ focal } \\
\text { stenosis at the distal It transverse sinus \& a pressure gradient } \\
\text { of } 11 \mathrm{~mm} \mathrm{Hg} \text { across this stenosis, which was later stented }\end{array}$ \\
\hline 3 & $40, \mathrm{~F}$ & $\begin{array}{l}\text { Venous sinus } \\
\text { stenosis }\end{array}$ & GETA & MRV & 3D-RV, MPR & $8^{*}$ & $\begin{array}{l}\text { Venous stenting; restriction of the cerebral venous drainage due } \\
\text { to severe stenosis of the rt transverse-sigmoid sinus junction } \\
\text { w/ pressure gradient of differential of } 18 \mathrm{~mm} \mathrm{Hg} \text { across the } \\
\text { stenotic portion; successful stenting of right transverse- } \\
\text { sigmoid junction w/ Precise } 8 \times 40-\mathrm{mm} \text { stent }\end{array}$ \\
\hline 4 & $62, \mathrm{~F}$ & $\begin{array}{l}\text { Venous sinus } \\
\text { stenosis }\end{array}$ & GETA & MRA & $\begin{array}{l}\text { 3D-RV, dual vol } \\
\text { w/ stent, MPR }\end{array}$ & $9^{*}$ & Venous stenting \\
\hline 5 & $63, \mathrm{~F}$ & $\begin{array}{l}\text { Lt asterion } \\
\text { meningioma }\end{array}$ & MAC & $\begin{array}{l}\text { T2WI } \\
\text { only }\end{array}$ & 3D-RV, MPR & $8^{*}$ & Lt transverse sinus focal stenosis \\
\hline 6 & $67, \mathrm{M}$ & $\begin{array}{l}\text { Lt parasagittal } \\
\text { vertex skull } \\
\text { lesion }\end{array}$ & MAC & MRI & $\begin{array}{l}\text { 3D-RV, bilat 3D- } \\
\text { 3D fusion }\end{array}$ & $10^{*}$ & $\begin{array}{l}\text { Narrowed but patent SSS w/ recruitment of a network of rt-sided } \\
\text { parasagittal cortical veins \& It vein of Trolard for additional } \\
\text { venous drainage }\end{array}$ \\
\hline 7 & $40, \mathrm{M}$ & $\begin{array}{l}\text { Rt parasagittal } \\
\text { meningioma }\end{array}$ & MAC & MRV & $\begin{array}{l}\text { 3D-RV, bilat 3D- } \\
\text { 3D fusion }\end{array}$ & $7^{*}$ & $\begin{array}{l}\text { Angiographic study showing patent SSS w/ trace flow anterior to } \\
\text { the tumor }\end{array}$ \\
\hline 8 & $65, \mathrm{M}$ & $\begin{array}{l}\text { Rt parasagittal } \\
\text { meningioma }\end{array}$ & MAC & MRV & 3D-RV & $8^{*}$ & Angiographic study showing patent SSS \\
\hline 9 & $33, \mathrm{~F}$ & $\begin{array}{l}\text { Posterior fossa } \\
\text { arachnoid cyst }\end{array}$ & MAC & MRV & 3D-RV, MPR & 8,10 & No intervention; straight sinus stenosis evaluation \\
\hline 10 & $34, \mathrm{M}$ & $\begin{array}{r}\text { Lt parietal para- } \\
\text { sagittal mass }\end{array}$ & MAC & MRV & $\begin{array}{l}\text { 3D-RV, bilat 3D- } \\
\text { 3D fusion }\end{array}$ & 9 & Embolized bilat middle meningeal artery feeders \\
\hline 11 & $22, \mathrm{~F}$ & Pulsatile tinnitus & GETA & CT & 3D-RV, MPR & 8 & Balloon-assisted coiling \\
\hline 12 & $29, \mathrm{~F}$ & Pulsatile tinnitus & GETA & CT & 3D-RV, MPR & 8 & Balloon-assisted coiling \\
\hline
\end{tabular}

GETA = general endotracheal anesthesia; MAC = monitored anesthesia care; MRA = MR angiography; $T 2 \mathrm{WI}=$ T2-weighted imaging.

* Radiography delay was not reported and was determined retrospectively.

\section{Discussion}

Imaging of cerebral veins receives less attention than that of arterial neurovasculature in the development of new angiographic imaging techniques. However, the findings obtained from these new techniques are of great importance and clinical relevance in many situations. Techniques and protocols that were initially developed for visualizing arterial structures can be modified to visualize venous structures and provide information that can be highly relevant clinically. We describe the application of $3 \mathrm{D}$ rotational angiography to venous structures (3D-RV) and MPR images in order to evaluate cerebral venous vasculature and its relationship to surrounding brain parenchyma and bony anatomy.

\section{Venous Diverticula}

The venous diverticula evaluated were certainly visible with DSA, but their relationship to the bone and surrounding opacified arteries obscured optimal visualization on DSA. In cases of arterial aneurysms, it is commonplace to perform 3D-RA especially for treatment planning and to obtain working views for intervention. Both patients in
Cases 11 and 12 underwent successful embolization with the use of 3D-RV in the same fashion. Previous reports have described imaging for these lesions using multidetector $\mathrm{CT}^{;} ;{ }^{6}$ the advantage of the presented 3D-RV allows diagnostic imaging and treatment to be done in the same setting, with the assistance of intraprocedural 3D guidance.

\section{Dura-Based Masses}

In most cases of dura-based masses, noninvasive imaging is equivocal as to the patency of the sinus, which is a key preoperative consideration. Three-dimensional RV and/or CTV-like reconstructions are sensitive tools in the assessment of sinus patency. For further assessment, 3D$3 \mathrm{D}$ fusion was performed in some cases (discussed below in $3 D-3 D$ Fusion).

\section{Technical Nuances}

In our experience, $3 \mathrm{D}-\mathrm{RV}$ is a versatile technique that can be applied in routine diagnostic angiography. The typical x-ray delay to allow contrast to flow into the venous system is generally $8-9$ seconds. This delay can be 

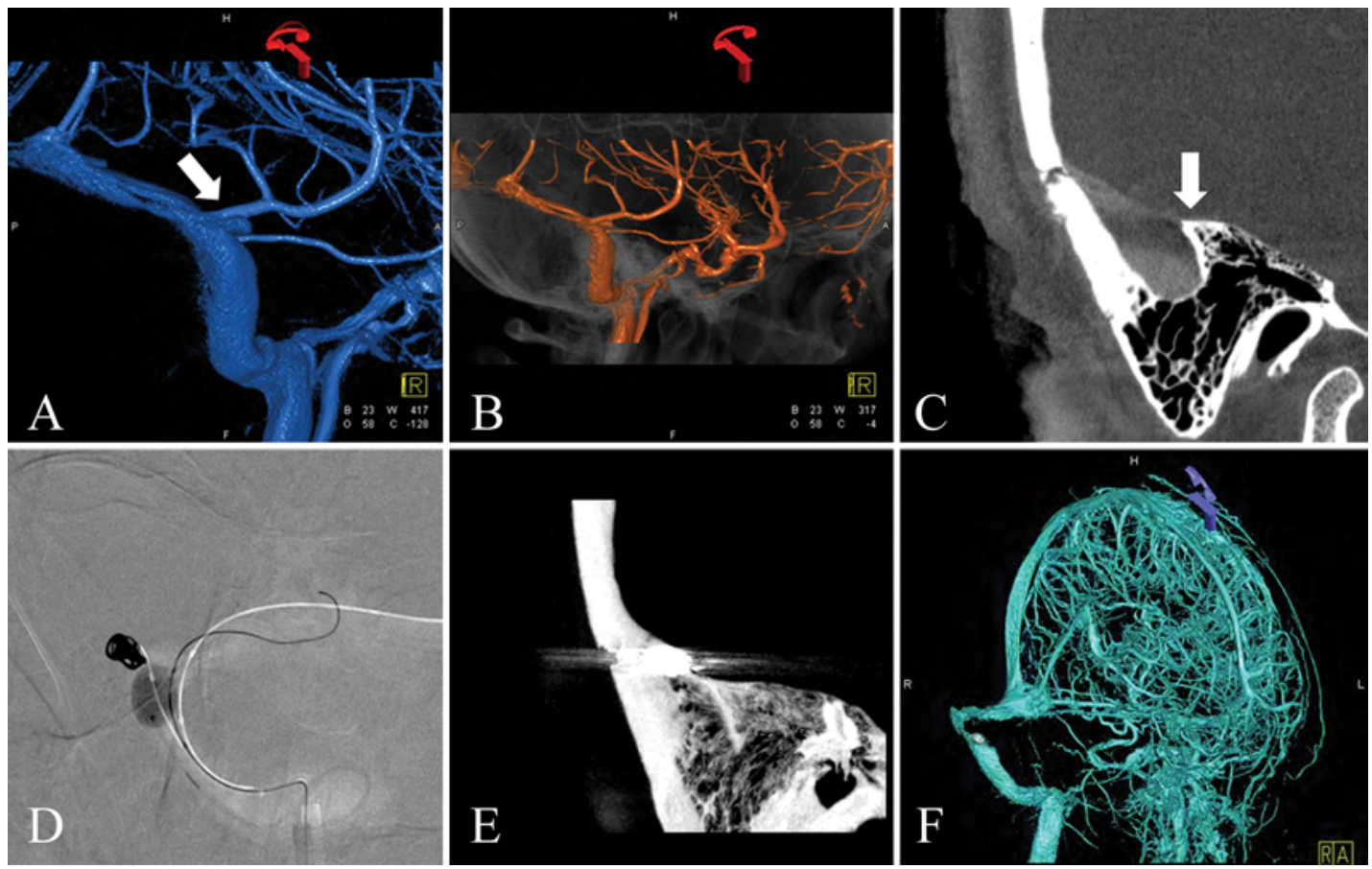

FIG. 2. Case 12. A: Three-dimensional RV image, showing a venous diverticulum/venous aneurysm (arrow). B: Lateral projection of the 3D-RV reconstruction, with dual-volume imaging showing the osseous anatomy, shows its location relative to the mastoid. C: The venous sac is seen projecting into the mastoid (arrow), explaining the symptoms of tinnitus. D: Intraprocedural image of the balloon-assisted coiling of the aneurysm. E and F: The diverticulum was fully obliterated with coils $(E)$, guided by the $3 \mathrm{D}-\mathrm{RV}$, with postprocedural dual-volume 3D-RV (F). Figure is available in color online only.
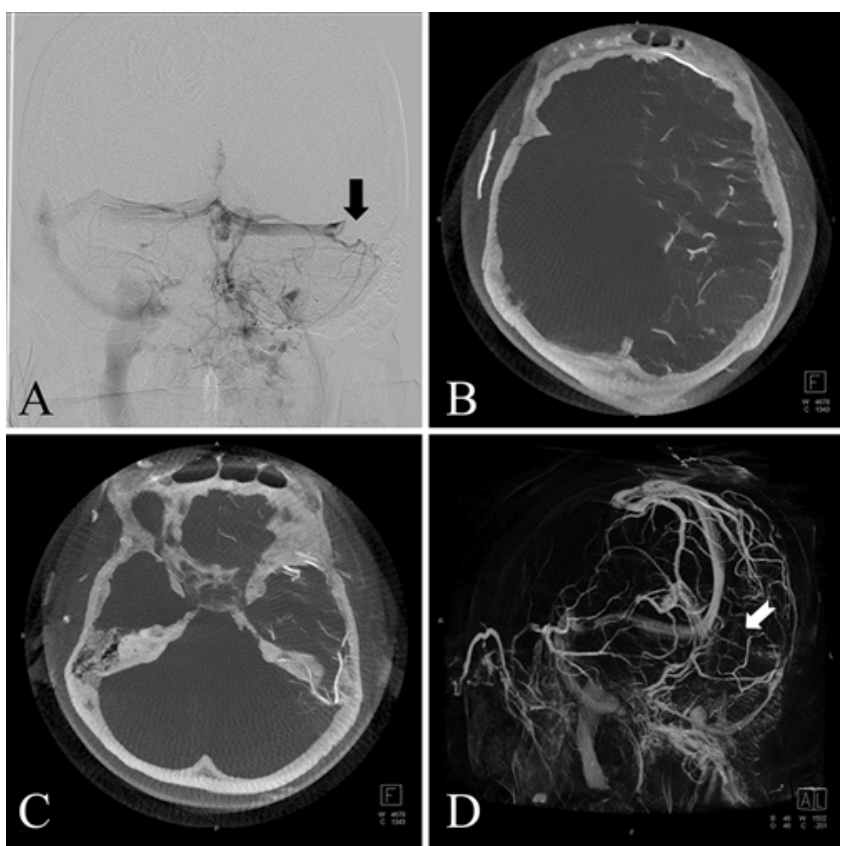

FIG. 3. Case 5. A: Anteroposterior DS angiogram showing occlusion of the lateral portion of the left transverse sinus (arrow). B: Axial "CTVlike" reconstruction showing a mass lesion in the region, along with the veins draped over the medial portion of the mass. C: The "CTV-like" images also showed the vein of Labbé draining just lateral to the area of occlusion, at the transverse-sigmoid junction. D: Three-dimensional RV showing the veins of the left hemisphere and the occluded left transverse sinus (arrow).
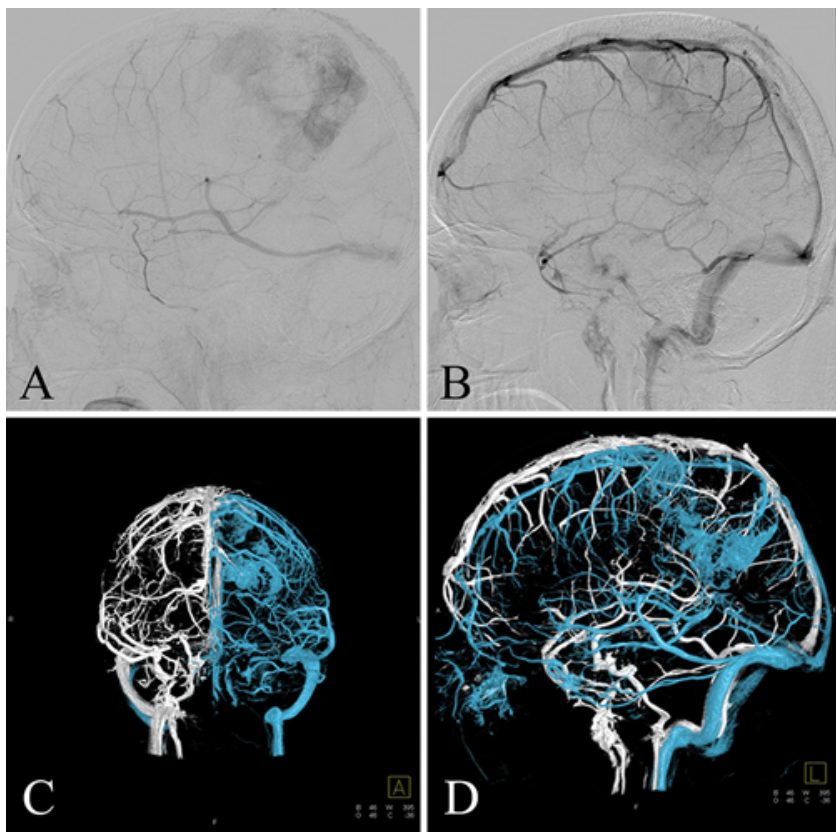

FIG. 4. Case 10. A: Late-arterial phase DS angiogram of the left hemisphere showing a tumor blush in the left parietal region. Flow through the posterior portion of the SSS was present. B: Late-venous phase DS angiogram of the right hemisphere, again showing flow in the posterior SSS, although there is no clear contiguity with the anterior portion of the sinus. C and D: Three-dimensional RV images from each side were fused in a "3D-3D fusion" technique, showing the small contributions to sinus flow from each side, confirming patency of the SSS across the region of tumor despite invasion. Figure is available in color online only. 


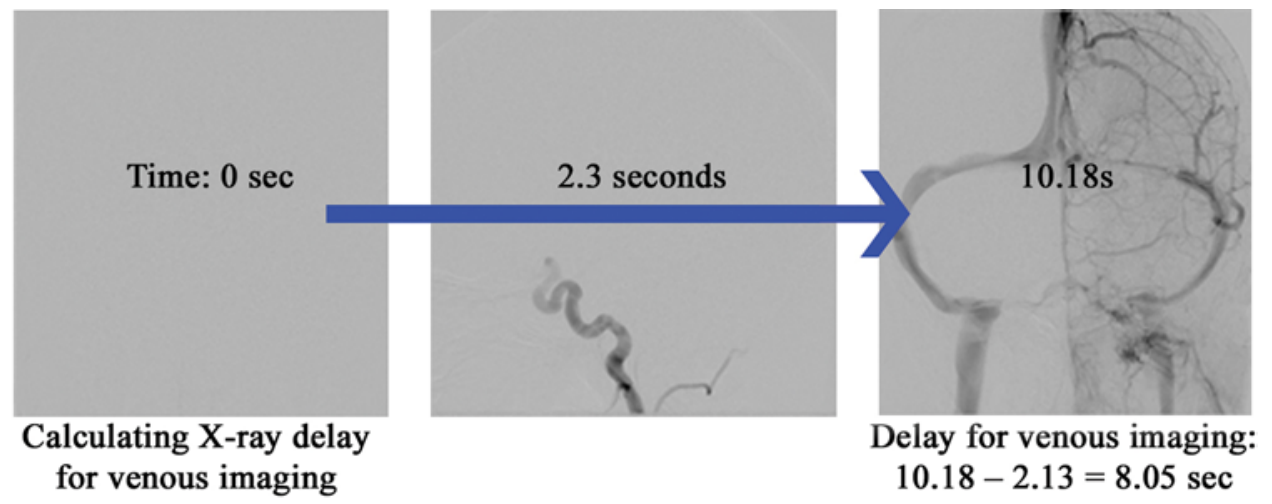

FIG. 5. The x-ray delay was set to the time difference between the acquisition frames corresponding to the arrival of contrast in the veins and the ICA. Figure is available in color online only.

calculated exactly for each patient using a 2D DSA image (Fig. 5). The other parameters for 3D-RV (bolus dose and timing) remain the same as those for 3D-RA. We recommend the use of an 8- to 9-second x-ray delay for most lesions, with the more accurate method of assessing maximal opacification being used only when necessary.

The effective breath hold/immobile time for the patient is the delay plus the 2 rotation times (10 seconds), resulting in a slightly longer breath hold compared with 3DRA. However, in our experience this can be done in most compliant patients. In patients who cannot comply with a longer breath hold, we recommend acquiring a single scan with patients holding their breath for only $4-5$ seconds during the acquisition. The images reconstructed in this setting must be processed and windowed appropriately to visualize the vasculature by itself. Furthermore, motioncompensated CBCT algorithms are also available to accurately reconstruct the vasculature to adjust for breathing between the scans. ${ }^{13}$

\section{Clinical Relevance}

There are many situations in which the venous system is of particular importance in neurosurgical pathologies, and a detailed understanding of the deep veins or sinuses can affect clinical decision making. In such situations, noninvasive imaging modalities such as MRV and CTV may be insufficiently sensitive. In cases such as the parasagittal meningiomas presented, understanding the location of venous occlusion and the presence of any trace flow can affect the decision to sacrifice the superior sagittal sinus, if necessary, in surgery. In cases of venous sinus stenosis (Case 4), a more accurate estimation of the degree of stenosis can affect the decision to treat altogether. The cases described in this report are quite varied, demonstrating the versatility of this technique. An example of this is in Case 4 , in which 2D DSA insufficiently demonstrated severe stenosis of the transverse sinus (Fig. 1A); the stenosis was observed on 3D-RV (Fig. 1D), but it was clearest on the MPR images (Fig. 1B and C). The techniques can be applied to fit the clinical situation.

\section{D-3D Fusion}

Postprocessing equipment allows the merge or "fu- sion" of 2 separate 3D volumes into 1 volume that can be manipulated. This 3D-3D fusion technique has been particularly useful for the anterior choroidal artery complex ${ }^{5}$ and when assessing for anterior cerebral artery anomalies (unpublished data). This can be applied to the venous system-specifically for the SSS, which receives contributory flow from both hemispheres. In 4 cases of parasagittal tumors invading or compressing the SSS, 3D-3D fusion has been helpful in cases of equivocal flow in that it provides confirmatory imaging of flow that may be unclear from individual DSA or unilateral 3D. An ideal example of this is in Case 10. This patient appeared to have an occluded SSS from the right injection; 3D-3D fusion of the bilateral ICA injections was performed and demonstrated patency of the sinus (Fig. 4). This could be applied to ICA-vertebral artery 3D-3D fusion as well in order to assess the posterior third of the SSS. The applications for this technique are rare, but it is a useful technique to keep in the arsenal nonetheless.

\section{Advantages Over Alternative Imaging Techniques}

For most standard clinical evaluations, CTV or MRV is usually sufficient. However, the quality of these studies varies among institutions and injection protocols. Thus, clinical situations occasionally arise that merit evaluation with diagnostic angiography. ${ }^{1}$ For these patients, who have undertaken the procedural risk and cost of an invasive procedure, the neurointerventionalist should gather sufficient data to make a confident interpretation of the vasculature using these advanced techniques. The techniques described are useful adjuncts to 2D DSA and solidify angiography as the gold standard in comparison with CTV and MRV. ${ }^{1}$ With timed x-ray delays, the opacification of the veins, thin slices, and improved reconstructions, 3D-RV and associated MPR images provide clear and reassuring visualization of the venous system and serve as an adjunct imaging modality for assessing cerebral venous anatomy. The marginal benefit of these techniques is highest in cases in which standard imaging is equivocal.

\section{Limitations}

The techniques of 3D-RV as described have several limitations and drawbacks. The most significant among 
these is that the quality of the subtracted 3D images as well as the MPR images depends on windowing and, at times, nuanced postprocessing. The potential for human error in adjusting images prior to review may lead to incorrect interpretation. Thus, whenever 3D-RV is used, we recommend that the interpreting neurointerventionalist be involved in postprocessing. Other drawbacks include the additional contrast load and radiation dose. The contrast bolus used in 3D-RV is the same as that used for 3D-RA $(2.5 \mathrm{ml} / \mathrm{sec}$ injection rate for a total volume of $18 \mathrm{ml})$. The radiation dose is similarly a marginal addition over the rest of the procedure and is equivalent to standard 3D-RA. Time is an additional factor to consider. Although use of this technique does not add more time, it does require additional time in postprocessing. Thus, we do not recommend its use in all cases, but we do recommend it for select, equivocal cases.

We illustrate here a proof of concept for the technique and some potential applications. Future studies will further assess the clinical utility and expand the applications to other venous lesions, and prove the value of these techniques over standard ones. Other potential applications include evaluation of cavernous sinus (Tolosa-Hunt syndrome), vein of Galen malformations, and venous aneurysms/varices. These advanced imaging techniques are meant to be an adjunct to 2D DSA, MRV, and CTV.

\section{Conclusions}

Three-dimensional RV can be used to assess the cerebral venous vasculature in a manner distinct from established modalities. We present the protocol and technique along with potential applications to venous sinus stenosis, venous diverticula, and tumors invading the venous sinuses.

\section{References}

1. Agid R, Shelef I, Scott JN, Farb RI: Imaging of the intracranial venous system. Neurologist 14:12-22, 2008

2. Busser WM, Arntz MJ, Jenniskens SF, Deinum J, Hoogeveen YL, de Lange F, et al: Image registration of cone-beam computer tomography and preprocedural computer tomography aids in localization of adrenal veins and decreasing radiation dose in adrenal vein sampling. Cardiovasc Intervent Radiol 38:993-997, 2015

3. Harmoush S, Chinnadurai P, El Salek K, Metwalli Z, Herce $\mathrm{H}$, Bhatt A, et al: Multimodality image-guided sclerotherapy of low-flow orbital vascular malformations: report of singlecenter experience. J Vasc Interv Radiol 27:987-995, 995.e1-995.e4, 2016

4. Honarmand AR, Gemmete JJ, Hurley MC, Shaibani A, Chaudhary N, Pandey AS, et al: Adjunctive value of intraarterial cone beam CT angiography relative to DSA in the evaluation of cranial and spinal arteriovenous fistulas. J Neurointerv Surg 7:517-523, 2015

5. Jalali A, Srinivasan VM, Chinnadurai P, Kan P, Arthur A, Duckworth EA: Two-color 3D-3D fusion of selective rotational cerebral angiograms: a novel approach to imaging in cerebrovascular neurosurgery. J Neurointerv Surg 8:10561060, 2016
6. Krishnan A, Mattox DE, Fountain AJ, Hudgins PA: CT arteriography and venography in pulsatile tinnitus: preliminary results. AJNR Am J Neuroradiol 27:1635-1638, 2006

7. Lee EW, So N, Chapman R, McWilliams JP, Loh CT, Busuttil RW, et al: Usefulness of intra-procedural cone-beam computed tomography in modified balloon-occluded retrograde transvenous obliteration of gastric varices. World J Radiol 8:390-396, 2016

8. Lucatelli P, Corona M, Argirò R, Anzidei M, Vallati G, Fanelli F, et al: Impact of 3D rotational angiography on liver embolization procedures: review of technique and applications. Cardiovasc Intervent Radiol 38:523-535, 2015

9. Matsumoto M, Kodama N, Sakuma J, Sato S, Oinuma M, Konno Y, et al: 3D-CT arteriography and 3D-CT venography: the separate demonstration of arterial-phase and venousphase on 3D-CT angiography in a single procedure. AJNR Am J Neuroradiol 26:635-641, 2005

10. Mitsuhashi Y, Nishio A, Kawahara S, Ichinose T, Yamauchi $\mathrm{S}$, Naruse H, et al: Morphologic evaluation of the caudal end of the inferior petrosal sinus using 3D rotational venography. AJNR Am J Neuroradiol 28:1179-1184, 2007

11. Nishio A, Takami T, Ohata K, Hara M, Mitsuhashi Y, Yokote $\mathrm{H}$, et al: Three-dimensional rotation venography using the digital subtraction angiography unit with a flat-panel detector: usefulness for the transtemporal/transtentorial approaches. Neuroradiology 46:876-882, 2004

12. Rangel-Castilla L, Krishna C, Klucznik R, Diaz O: Endovascular embolization with Onyx in the management of sinus pericranii: a case report. Neurosurg Focus 27(5):E13, 2009

13. Sisniega A, Stayman JW, Cao Q, Yorkston J, Siewerdsen JH, Zbijewski W: Image-based motion compensation for highresolution extremities cone-beam CT. Proc SPIE Int Soc Opt Eng 9783:97830K, 2016

14. Srinivasan VM, Schafer S, Ghali MG, Arthur A, Duckworth EA: Cone-beam CT angiography (Dyna CT) for intraoperative localization of cerebral arteriovenous malformations. $\mathbf{J}$ Neurointerv Surg 8:69-74, 2016

15. Srivatanakul K, Osada T, Aoki R, Sorimachi T, Matsumae M: Transvenous embolization of cavernous sinus dural arteriovenous fistula through a thrombosed inferior petrosal sinus utilizing 3D venography. Interv Neuroradiol 21:362-365, 2015

\section{Disclosures}

Gouthami Chintalapani reports being an employee of Siemens Medical Solutions USA, Inc.

\section{Author Contributions}

Conception and design: Kan, Srinivasan. Acquisition of data: all authors. Analysis and interpretation of data: Kan, Srinivasan, Chintalapani. Drafting the article: Kan, Srinivasan, Chintalapani. Critically revising the article: all authors. Reviewed submitted version of manuscript: Kan, Srinivasan, Chintalapani. Administrative/technical/material support: Chintalapani. Study supervision: Kan, Duckworth.

\section{Correspondence}

Peter Kan, Department of Neurosurgery, Baylor College of Medicine, 7200 Cambridge St., Ste. 9A, Houston, TX 77030. email: peter.kan@bcm.edu. 